\title{
Nonlinear anomalous Hall effect and negative magnetoresistance in a system with random Rashba field
}

\author{
V. K. Dugaev ${ }^{1,2,3}$, M. Inglot ${ }^{1}$, E. Ya. Sherman ${ }^{4,5}$, J. Berakdar, ${ }^{2}$ and J. Barnaś $6{ }^{6,7}$ \\ ${ }^{1}$ Department of Physics, Rzeszów University of Technology, \\ al. Powstańców Warszawy 6, 35-959 Rzeszów, Poland \\ ${ }^{2}$ Institut für Physik, Martin-Luther-Universität Halle-Wittenberg, \\ Heinrich-Damerow-Str. 4, 06120 Halle, Germany \\ ${ }^{3}$ Department of Physics and CFIF, Instituto Superior Técnico, \\ TU Lisbon, Av. Rovisco Pais, 1049-001 Lisbon, Portugal \\ ${ }^{4}$ Department of Physical Chemistry, Universidad del País Vasco UPV-EHU, 48080, Bilbao, Spain \\ ${ }^{5}$ IKERBASQUE Basque Foundation for Science, Alameda Urquijo 36-5, 48011 Bilbao, Spain \\ ${ }^{6}$ Faculty of Physics, Adam Mickiewicz University, Umultowska 85, 61-614 Poznań, Poland \\ ${ }^{7}$ Institute of Molecular Physics, Polish Academy of Sciences, Smoluchowskiego 17, 60-179 Poznań, Poland
}

(Dated: April 22, 2018)

\begin{abstract}
We predict two spin-dependent transport phenomena in two-dimensional electron systems, which are induced by spatially fluctuating Rashba spin-orbit interaction. When the electron gas is magnetized, the random Rashba interaction leads to the anomalous Hall effect. An example of such a system is a narrow-gap magnetic semiconductor-based symmetric quantum well. We show that the anomalous Hall conductivity reveals a strongly nonlinear dependence on the magnetization, decreasing exponentially at large spin density. We also show that electron scattering from a fluctuating Rashba field in a two-dimensional nonmagnetic electron system leads to a negative magnetoresistance arising solely due to spin-dependent effects.
\end{abstract}

PACS numbers: 72.25.Dc, 73.23.-b, 73.50.Bk

Introduction. Effects of electron spin on charge transport, being the basic idea of spintronics, attract a great deal of interest in fundamental and applied physics as well as in materials science. There are several origins of the spin dependence of charge transport; one of them is the spin-orbit ( $\mathrm{SO}$ ) interaction. This interaction also plays an important role in spin manipulation in spintronics devices [1-4]. Of particular importance is the Rashba SO coupling, usually attributed to quasi twodimensional (2D) electron systems in metallic or semiconductor nanolayers on a substrate, 2D semiconductor heterostructures with no $z \rightarrow-z$ symmetry (with the axis $z$ normal to the layer), or to surface states [5 7]. This interaction enables an electrical control of spin precession of $2 \mathrm{D}$ electrons - the phenomenon utilized in still hypothetical Datta-Das transistor [8], where current depends on the angle of spin precession when carriers pass through the device.

Important manifestations of the SO coupling are the anomalous Hall effect (AHE) and spin Hall effect (SHE) [9 11]. In the case of AHE, a charge current perpendicular to electric field appears without external magnetic field when the system has a nonzero spontaneous magnetization [12]. In the SHE, in turn, static electric field generates spin current perpendicular to the field orientation. Both effects, however, can be completely suppressed by disorder 13 15].

In symmetrical semiconductor quantum wells, the Rashba SO interaction vanishes on average. However, spatial fluctuations of the Rashba coupling may still appear in the system and can play a qualitatively important role [16 18]. Surprisingly, in the case of spatially fluctuating Rashba field, the SHE in 2D electron gas becomes robust to the effect of impurities, i.e., there is no complete suppression of the spin-Hall conductivity by disorder [19].

In this Letter we predict further two manifestations of spin-dependent transport in 2D electron systems with random Rashba interaction. First, we show that systems with homogeneous magnetization display AHE. More specifically, we calculate the off-diagonal conductivity of a 2D magnetized electron gas in a symmetric quantum well with spatially correlated fluctuations of SO interaction. We show that the Hall conductivity reveals a very unusual dependence on the magnetization: it reaches a maximum followed by a fast decrease to zero for magnetization increasing further. Second, we calculate the magnetoresistivity of a nonmagnetic electron gas for a magnetic field parallel to the quantum well and show that the magnetoresistance is negative. This negative magnetoresistance (NMR) is a purely classical effect, unrelated to quantum localization corrections to the conductivity. In both above mentioned phenomena, the random spin-orbit coupling is crucial and either generates a macroscopic electric current or modifies the macroscopic conductivity.

Model. The generic model of a 2D electron gas with random Rashba interaction is described by the Hamiltonian $\hat{H}=\hat{H}_{0}+\hat{H}^{\text {(so) }}$, where (we use the units with $\hbar \equiv 1)$

$$
\hat{H}_{0}=-\frac{\nabla^{2}}{2 m}+U_{\mathrm{rnd}}-M \sigma_{z}+\sigma_{x} \beta B
$$




$$
\hat{H}^{(\mathrm{so})}=-\frac{i}{2} \sigma_{x}\left\{\nabla_{y}, \lambda(\mathbf{r})\right\}+\frac{i}{2} \sigma_{y}\left\{\nabla_{x}, \lambda(\mathbf{r})\right\} .
$$

Here $M$ is half of the spin splitting corresponding to the homogeneous magnetization along the axis $z$, having purely spin character and not related to any conventional macroscopic magnetic field [15]. The random Rashba coupling parameter $\lambda(\mathbf{r})$ (for $\mathbf{r}=(x, y)$ ) has zero average, $\langle\lambda(\mathbf{r})\rangle=0$, and a Gaussian correlator $\left\langle\lambda(\mathbf{r}) \lambda\left(\mathbf{r}^{\prime}\right)\right\rangle$, while $\sigma_{i}(i=x, y, z)$ are the spin Pauli matrices and $m$ is the electron effective mass. The term $U_{\text {rnd }}$ describes the spin-independent disorder assumed to be of white-noise type, $B$ is the in-plane magnetic field along the axis $x$, and $\beta=g \mu_{B} / 2$, where $g$ is the electron Landé factor. Assuming in-plane magnetic field we avoid the diamagnetic orbital effects, and therefore we take into account only the Zeeman term in Eq.(1). The magnetization leads to splitting of the Fermi surface, and in the limit of small magnetization $\Delta K \equiv k_{F \uparrow}-k_{F \downarrow}=2 M m / k_{F}$, as shown in Fig. 1, where $k_{F}$ is the Fermi wavevector in the corresponding nonmagnetic state.

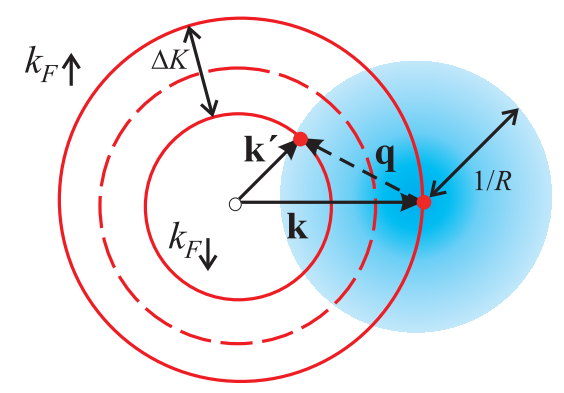

FIG. 1: Spin-split Fermi surface. $1 / R$ is the possible range of momentum change, $|\mathbf{q}|=\left|\mathbf{k}^{\prime}-\mathbf{k}\right|$ in the spin-flip scattering process between two Fermi surfaces. Dashed circle corresponds to the Fermi momentum $k_{F}$. In the limit of small Fermi surface splitting, $\Delta K=2 M m / k_{F}$ for $B=0$ (spin orientation is taken with respect to the $z$-axis), or $|\Delta K|=2 \beta B m / k_{F}$ for $M=0$ (spin orientation is taken with respect to the $x$-axis).

In the momentum representation Eq. (2) can be rewritten as

$$
H_{\mathbf{k} \mathbf{k}^{\prime}}^{(\mathrm{so})}=\frac{\lambda_{\mathbf{k k}^{\prime}}}{2}\left[\sigma_{x}\left(k_{y}+k_{y}^{\prime}\right)-\sigma_{y}\left(k_{x}+k_{x}^{\prime}\right)\right],
$$

where $k_{i}\left(k_{i}^{\prime}\right)$ are the in-plane momentum components $(i=x, y)$. For an external electromagnetic field, $\mathbf{A}(t)=$ $\mathbf{A}_{0} e^{-i \omega t}$, one has to make the following replacement in Eqs. (11) and (2): $\nabla \rightarrow \nabla-i e \mathbf{A} / c$. Thus, the matrix elements of the coupling to the electromagnetic field take the form

$$
\begin{aligned}
H_{\mathbf{k k}^{\prime}}^{(A)}= & {\left[-\frac{e}{m c}(\mathbf{k} \cdot \mathbf{A})+\frac{e^{2}}{2 m c^{2}} A^{2}\right] \delta_{\mathbf{k k}^{\prime}} } \\
& -\frac{e}{c} \lambda_{\mathbf{k k}^{\prime}}\left(\sigma_{x} A_{y}-\sigma_{y} A_{x}\right)
\end{aligned}
$$

and include the term following from the random Rashba interaction. Correspondingly, the matrix elements of the charge current operator $\hat{\mathbf{j}}=-c \partial \hat{H}^{(A)} / \partial \mathbf{A}$ are

$$
\left(j_{x, y}\right)_{\mathbf{k k}^{\prime}}=\frac{e}{m}\left(k_{x, y}-\frac{e}{c} A_{x, y}\right) \delta_{\mathbf{k k}^{\prime}} \mp e \lambda_{\mathbf{k k}^{\prime}} \sigma_{y, x} .
$$

The random spin-orbit coupling leads to spin-flip scattering between states with opposite spin orientations. This, in turn, results in two effects to be analyzed below: the AHE and the NMR.

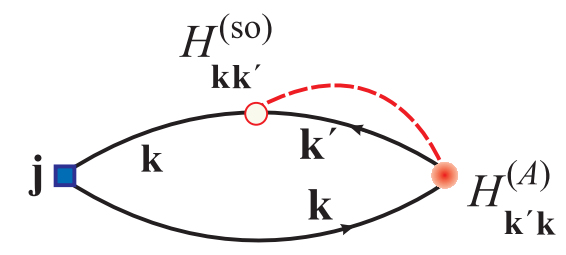

FIG. 2: Feynman graph for the anomalous Hall current. Matrix elements in the vertices are determined by Eqs.(3)-(15).

Anomalous Hall effect. Let us begin with the AHE induced by fluctuating Rashba field. Hence, we put here $B=0$, assume an electric field $\mathbf{E}$ along the axis $y$, and consider charge current normal to the field. Upon calculating the off-diagonal linear conductivity [20], we find that it can be written as the difference of the contributions from two different spin channels,

$$
\sigma_{x y}=\sigma_{x y \uparrow}-\sigma_{x y \downarrow} .
$$

In the approximation corresponding to the loop diagrams shown in Fig. 2 one finds $\left(s \neq s^{\prime}\right)$

$$
\begin{aligned}
\sigma_{x y, s}= & \frac{e^{2}}{m} \sum_{\mathbf{k q}}\left|\lambda_{q}\right|^{2} k_{y}\left(2 k_{y}-q_{y}\right) \delta\left(\mu-\varepsilon_{k-q, s^{\prime}}\right) \\
& \times G_{k, s}^{R} G_{k, s}^{A}
\end{aligned}
$$

where $G_{k, s}^{R}$ and $G_{k, s}^{A}(s=\uparrow, \downarrow)$ are the retarded and advanced Green functions, respectively. Due to scattering from the fluctuating SO field, electrons with opposite spins are turned to the opposite transverse directions, so $\sigma_{x y \uparrow}$ and $\sigma_{x y \downarrow}$ enter Eq. (6) with opposite signs (they are added in the case of spin Hall effect). The resulting AHE for $M \neq 0$ is nonzero due to the spin polarization (magnetization) of the electron gas.

Upon calculating the sum over $k$ in Eq. (7) one obtains

$$
\begin{aligned}
\sigma_{x y \uparrow, \downarrow}= & \frac{2 e^{2} m \nu \tau_{\uparrow, \downarrow}}{\pi k_{F \uparrow, \downarrow}} \int_{0}^{\infty} d q\left(\mu-\frac{q^{2}}{8 m} \pm \frac{3 M}{2}\right)\left|\lambda_{q}\right|^{2} \\
& \times \int_{0}^{\pi} d \varphi \delta\left(\cos \varphi-\frac{q}{2 k_{F \uparrow, \downarrow}} \mp \frac{2 m M}{k_{F \uparrow, \downarrow} q}\right),
\end{aligned}
$$

where $\tau_{\uparrow, \downarrow}$ is the spin-dependent relaxation time, $\nu$ is the density of states per spin, and the upper (lower) sign corresponds to spin-up (spin-down) electrons. The spindependence of the relaxation time $\tau_{\uparrow, \downarrow}$ can result from the 


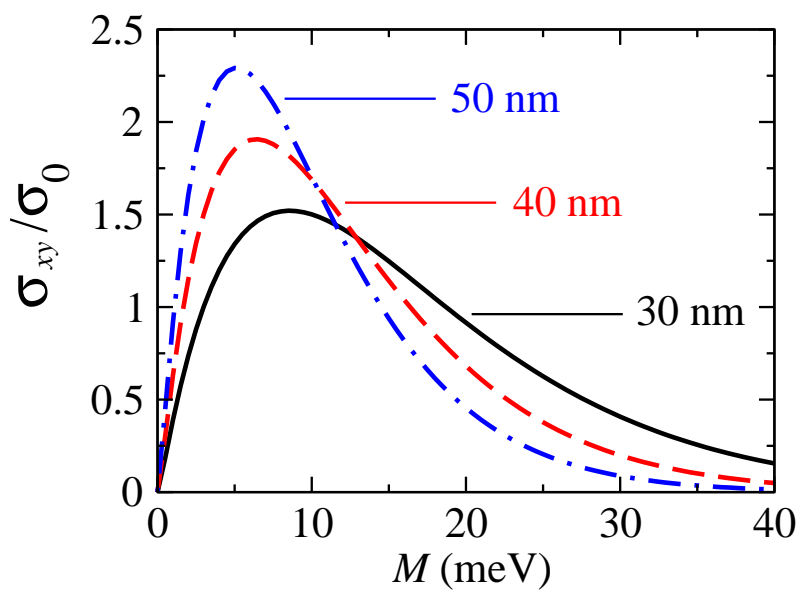

FIG. 3: Anomalous Hall conductivity as a function of spin splitting parameter $M$ for indicated values of $R$. Other parameters are defined in the text.

spin dependence of the corresponding Fermi momentum. In the case of white-noise short range disorder, $\tau$ is determined solely by the density of states at the Fermi level, and, therefore, does not depend on the spin orientation.

If the magnetization is relatively weak, $\sqrt{2 m M}<k_{F}$, both spin subbands are occupied and the spin-projected conductivity is

$$
\begin{aligned}
& \sigma_{x y \uparrow, \downarrow}=\frac{2 e^{2} m \nu \tau_{\uparrow, \downarrow}}{\pi k_{F \uparrow, \downarrow}} \\
& \times \int_{\Delta K}^{k_{F \uparrow}+k_{F \downarrow}} d q\left|\lambda_{q}\right|^{2} \frac{\mu-q^{2} / 8 m \pm 3 M / 2}{\sqrt{1-\left(q / 2 k_{F \uparrow, \downarrow} \pm 2 m M / k_{F \uparrow, \downarrow}\right)^{2}}} .
\end{aligned}
$$

In turn, when the magnetization is sufficiently strong, $\sqrt{2 m M}>k_{F}$, only one spin subband is occupied. Spinflip scattering is then absent and $\sigma_{x y}$ vanishes. As we show below in more details, dependence of the AHE on the magnetization $M$ is rather unusual.

For further calculations we need a more specific Rashba field correlator, and we assumed it in the following generic form: 18]

$$
\left|\lambda_{q}\right|^{2}=2 \pi\left\langle\lambda^{2}\right\rangle R^{2} e^{-q R} .
$$

Here $\left\langle\lambda^{2}\right\rangle$ characterizes the field variation, and the correlation length $R$ is of the order of the distance between the quantum well and the dopant layers since the in-plane distribution of electric field in the well is controlled by this parameter 21].

Using Eqs. (10) and (6), we have calculated numerically the Hall conductivity as shown in Fig. 3. The conductivity is presented there in the units of $\sigma_{0}=$ $\sigma_{D}\left\langle\lambda^{2}\right\rangle k_{F}^{2} / E_{F}^{2}$, where $\sigma_{D}=n e^{2} \tau / m$ is the Drude conductivity, and $E_{F}=k_{F}^{2} / 2 m$. We used the parameters characteristic for InSb: effective mass $m=0.0134 m_{0}$, and electron density $n=5 \times 10^{11} \mathrm{~cm}^{-2}$ related to the
Fermi momentum $k_{F}=(2 \pi n)^{1 / 2}$. We can estimate the ratio of $\sigma_{0} / \sigma_{D}$ by using the relation between the electric field $\mathcal{E}$ and the spin-orbit coupling in the form $\left\langle\lambda^{2}\right\rangle=$ $\xi^{2} e^{2}\left\langle\mathcal{E}_{r}^{2}\right\rangle$, where the variation in the random electric field $\left\langle\mathcal{E}_{r}^{2}\right\rangle=2 \pi e^{2} n_{d} / \kappa^{2} R^{2}$. For InSb $\xi \approx 5 \mathrm{~nm}^{2},[22,23]$ and for typical parameters of quantum wells we obtain $\sqrt{\left\langle\lambda^{2}\right\rangle}$ of the order of $10^{-6} \mathrm{meVcm}$ and $\sigma_{0} / \sigma_{D}$ of the order of $10^{-2}-10^{-1}$. Thus, the maximum anomalous Hall conductivity can be of an order of 0.01-0.1 of the Drude conductivity.

As follows from Fig. 3, the Hall conductivity has a sharp maximum at a certain value of the parameter $M$. The physical reason for such a behavior is that the spinflip scattering from fluctuating Rashba coupling is effective only for a relatively small change in electron momentum, $q<1 / R$. Thus, if $\Delta K>1 / R$, these elastic spin-flip processes become suppressed and the AHE vanishes. To have a better physical insight into the problem, we present the Hall conductivity in an approximate form as

$$
\frac{\sigma_{x y}}{\sigma_{0}} \sim k_{F}^{2} R^{2} \frac{M}{E_{F}} \exp \left(-\frac{M}{E_{F}} k_{F} R\right) .
$$

At zero $M$ the contributions from different spins exactly compensate each other, while with the increase in $M$ to the region where $\Delta K R=\left(M / E_{F}\right) k_{F} R \gg 1$, the spinflip transitions are suppressed and the Hall conductivity vanishes as well. The maximum of conductivity is achieved for $M=E_{F} / k_{F} R$. The resulting maximum anomalous Hall conductivity is then of the order

$$
\max \left(\frac{\sigma_{x y}}{\sigma_{0}}\right) \sim k_{F} R / e,
$$

where $e \approx 2.718 \ldots$ is the base if the natural logarithm.

It is instructive to compare the anomalous and the conventional Hall conductivities. Magnetization of the order of $E_{F} / k_{F} R$ for InSb with the $g$-factor close to 50 can be achieved in the fields $B_{z}$ of the order of $1 \mathrm{~T}$. The ratio of the Drude and Hall conductivity is $\omega_{c} \tau$, where $\omega_{c}$ is the cyclotron frequency. In the field of $1 \mathrm{~T}$ for a sample with the mobility of $10^{5} \mathrm{~cm}^{2} / \mathrm{Vs}$, one finds $\omega_{c} \tau \approx 10$. As a result, the anomalous Hall conductivity can be of an order of $0.1-1.0$ of the conventional Hall conductivity. It can be extracted from the conventional Hall measurements taking into account an unusual dependence on the external magnetic field.

Negative magnetoresistance and anisotropic conductivity. We have shown above that the spin-flip scattering from fluctuating spin-orbit field leads to AHE in a magnetized electron gas. Now we demonstrate that such scattering also modifies the diagonal conductivity as the electron relaxation rate includes generally not only the term due to scattering from impurities but also the contribution from spin-flip scattering due to fluctuating Rashba coupling. Since the spin-flip scattering is essential at 
small spin splitting and decreases at large splitting, its contribution to spin relaxation time strongly depends on the spin polarization of the electron gas. This spin polarization can be modified by an external magnetic field, which in turn leads to the magnetoresistance. For this effect it is sufficient to consider the situation when the band splitting is not related to the homogeneous magnetization but is due to the Zeeman field. Therefore we consider now a nonmagnetic electron gas, $M=0$, and assume a nonzero magnetic field along the $x$ axis. We show below that spin-orbit fluctuating Rashba field leads then to a NMR.

To calculate the diagonal component of conductivity tensor we can use the kinetic equation, which takes into account spin-conserving and spin-flip transitions,

$$
\begin{aligned}
\frac{e}{m}(\mathbf{E} \cdot \mathbf{k}) \frac{\partial f_{\mathbf{k} \uparrow}^{0}}{\partial \varepsilon_{k}}= & -\sum_{\mathbf{k}^{\prime}}\left[W_{\mathbf{k k}^{\prime}}\left(f_{\mathbf{k} \uparrow}-f_{\mathbf{k}^{\prime} \uparrow}\right)\right. \\
& \left.+W_{\mathbf{k k}^{\prime}}^{f}\left(f_{\mathbf{k} \uparrow}-f_{\mathbf{k}^{\prime} \downarrow}\right)\right],
\end{aligned}
$$

and a similar equation for the opposite spin orientation. Here we introduced the notation

$$
\begin{aligned}
& W_{\mathbf{k k}^{\prime}}=2 \pi\left[w_{\mathbf{k k}^{\prime}}+\left|\lambda_{\mathbf{k k}^{\prime}}\right|^{2}\left(k_{y}+k_{y}^{\prime}\right)^{2}\right] \delta\left(\varepsilon_{k}-\varepsilon_{k^{\prime}}\right) \\
& W_{\mathbf{k k}^{\prime}}^{f}=2 \pi\left|\lambda_{\mathbf{k k}^{\prime}}\right|^{2}\left(k_{x}+k_{x}^{\prime}\right)^{2} \delta\left(\varepsilon_{k}-\varepsilon_{k^{\prime}} \pm 2 \beta B\right)
\end{aligned}
$$

for spin-conserving and spin-flip transitions, respectively, where $w_{\mathbf{k k}^{\prime}}$ corresponds to the spin-conserving scattering from usual disorder. If the potential of impurities is short range, $w_{\mathbf{k k}^{\prime}}$ can be taken independent on the momentum, $w_{\mathbf{k k}^{\prime}} \simeq w_{0}$. Although the solution of Eq. 113. can in general be presented as a spin-dependent sum of cylindrical Fermi surface harmonics, it cannot be solved in the general form due to anisotropy of matrix elements in Eqs. 114), 15) and the presence of two Fermi surfaces. However, for $k_{F} R \gg 1$, where one can with a high accuracy put $k_{x}=k_{x}^{\prime}$ and $k_{y}=k_{y}^{\prime}$, they can be greatly simplified for the electric field $\mathbf{E}$ parallel and perpendicular to the magnetic field $\mathbf{B}$.

Let us start with the limit of weak magnetic field, when the Zeeman splitting is very small, $\Delta K \ll 1 / R$. The spin-split Fermi surfaces are then almost identical, and can be considered as a single surface. The corresponding isotropic conductivity, $\sigma=\sigma_{D}+\delta \sigma^{\text {so }}$, can be also presented as $\sigma(B \rightarrow 0)=n e^{2} \tau_{\text {tot }} / m$, where $1 / \tau_{\text {tot }}=1 / \tau+1 / \tau_{\text {so }}$ includes the transport scattering rate $1 / \tau_{\text {so }}$ related to Eqs. (15) (spin-flip processes) and (14) (spin-conserving processes caused by spin-orbit coupling). As a result, $\delta \sigma^{\text {so }}=-\tau \sigma_{D} / \tau_{\text {so }}$. The rate $1 / \tau_{\text {so }}$ can be evaluated as $1 / \tau_{\text {so }} \sim 4\left\langle\lambda^{2}\right\rangle / v_{F} R$ and is of the order of $10^{11} \mathrm{~s}^{-1}$, leading to the negative correction of the order of $0.1 \sigma_{D}$ to the Drude conductivity at mobility $10^{5} \mathrm{~cm}^{2} / \mathrm{Vs}$.

When the magnetic field increases, the spin-flip processes become suppressed, as can be qualitatively seen in Fig 1, while spin-conserving ones remain almost intact.
In the limit of strong field, $\beta B m R \gg 1$, the spin-flip term, Eq. (15), vanishes and the conductivity is larger than at $B=0$ for both $\mathbf{E} \| \mathbf{B}$ and $\mathbf{E} \perp \mathbf{B}$ geometries. At the same time, as we see from (14), the remaining spinconserving processes include scattering from the random Rashba field that strongly depends on the orientation of momenta $\mathbf{k}$ and $\mathbf{k}^{\prime}$. As a result, the total scattering at large field becomes anisotropic. Solving the kinetic equation for this case we find that the conductivity at large $B$ is also anisotropic with $|\sigma(\mathbf{E} \| \mathbf{B})-\sigma(\mathbf{E} \perp \mathbf{B})|=$ $\left|\delta \sigma^{\text {so }} / 2\right|$, and the degree of anisotropy is of the order of several percent.

Note that the NMR effect we consider here is qualitatively different from the one of Ref. [24] for systems with long- and short-range disorder. The mechanism proposed by us has a solely spin-related origin and is not related to the orbital motion considered in Ref. [24]. Recent experiments [25] showed that the NMR cannot be fully explained in terms of the model of Ref. 24] and some spinrelated effects can be involved in the physics of this phenomenon. Moreover, our effect is purely classical and not related to NMR in the weak localization regime. 26, 27]

Summary. We have calculated the anomalous Hall conductivity and the magnetoresistance of $2 \mathrm{D}$ electron systems due to scattering from spatial fluctuations of Rashba spin-orbit interaction. The materials where this interaction can be essential are symmetric narrow-gap semiconductor quantum wells. One of the usual characterization methods for these materials is based on the measurements of AHE under the assumption that AHE is proportional to magnetization. This proportionality, however, is completely destroyed when the AHE is related to the fluctuating Rashba field. It should be stressed that the proposed mechanism of AHE can be important when the usual impurity scattering within the quantum well is small. In the case of nonmagnetic semiconductors it can be easily realized by donor impurities outside the quantum well. We have also demonstrated that the scattering of electrons from the fluctuations of Rashba field in nonmagnetic symmetric semiconductor quantum wells leads to a negative magnetoresistance. This effect can be realized for example in InSb or other narrow gap semiconductors, in which the Zeeman splitting and spin-orbit coupling are both very strong.

This work is partly supported by the DFG in Germany and by National Science Center in Poland as a research project in years 2011-2014 and the project DEC2012/04/A/ST3/00372. The work of EYS was supported by the MCINN of Spain grant FIS 2009-12773-C02-01, "Grupos Consolidados UPV/EHU del Gobierno Vasco" grant IT-472-10, and by the UPV/EHU under program UFI 11/55. 
[1] I. Zutić, J. Fabian, and Das Sarma, Rev. Mod. Phys. 76, 323 (2004).

[2] J. Fabian, A. Matos-Abiague, C. Ertler, P. Stano, and I. Zutic, Acta Phys. Slovaca 57, 565 (2007).

[3] M. W. Wu, J. H. Jiang, and M. Q. Weng, Phys. Rep. 493, 61 (2010).

[4] Spin Physics in Semiconductors, edited by M. I. Dyakonov (Springer, Berlin, 2008).

[5] E. I. Rashba, Sov. Phys. Solid State 2, 1874 (1964); Yu. A. Bychkov and E. I. Rashba, JETP Lett. 39, 79 (1984).

[6] P. Pfeffer and W. Zawadzki, Phys. Rev. B 59, R5312 (1999).

[7] T. Koga, J. Nitta, T. Akazaki, and H. Takayanagi, Phys. Rev. Lett. 89, 046801 (2002).

[8] S. Datta and B. Das, Appl. Phys. Lett. 56, 665 (1990).

[9] T. Jungwirth, Q. Niu, and A. H. MacDonald, Phys. Rev. Lett. 88, 207208 (2002); D. Culcer, A. MacDonald, and Q. Niu, Phys. Rev. B 68, 045327 (2003).

[10] M. Onoda and N. Nagaosa, J. Phys. Soc. Jpn. 71, 19 (2002); Phys. Rev. Lett. 90, 206601 (2003); Z. Fang, N. Nagaosa, K. S. Takahashi, A. Asamitsu, R. Mathieu, T. Ogasawara, H. Yamada, M. Kawasaki, Y. Tokura, and K. Terakura, Science 302, 92 (2003).

[11] J. Sinova, D. Culcer, Q. Niu, N. A. Sinitsyn, T. Jungwirth, and A. H. MacDonald, Phys. Rev. Lett. 92, 126603 (2004).

[12] V. K. Dugaev, A. Crépieux, and P. Bruno, Phys. Rev. B 64, 104411 (2001), and references therein.

[13] T. S. Nunner, N. A. Sinitsyn, M. F. Borunda, V. K. Dugaev, A. A. Kovalev, Ar. Abanov, C. Timm, T. Jungwirth, J. Inoue, A. H. MacDonald, and J. Sinova, Phys. Rev. B 76, 235312 (2007).

[14] H. A. Engel, E. I. Rashba, and B. I. Halperin, In: Handbook of Magnetism and Advanced Magnetic Materials, edited by H. Kronmüller and S. Parkin. Vol. 5: Spintronics and Magnetoelectronics. (John Wiley \& Sons, New
York, 2007).

[15] N. Nagaosa, J. Sinova, S. Onoda, A. H. MacDonald, and N. P. Ong, Rev. Mod. Phys. 82, 1539 (2010).

[16] E. Ya. Sherman, Appl. Phys. Lett. 82, 209 (2003); Phys. Rev. B 67, 161303 (2003).

[17] V. K. Dugaev, E. Ya, Sherman, V. I. Ivanov, and J. Barnaś, Phys. Rev. B 80, 081301(R) (2009).

[18] M. M. Glazov, E. Ya. Sherman, and V. K. Dugaev, Physica E 42, 2157 (2010).

[19] V. K. Dugaev, M. Inglot, E. Ya. Sherman, and J. Barnaś, Phys. Rev. B 82, 121310(R) (2010).

[20] A. A. Abrikosov, L. P. Gorkov, and I. E. Dzyaloshinski, Methods of Quantum Field Theory in Statistical Physics (Dover, New York, 1963).

[21] T. Ando, A. B. Fowler, and F. Stern, Rev. Mod. Phys. 54, 437 (1982).

[22] R. Winkler, Spin-Orbit Coupling Effects in TwoDimensional Electron and Hole Systems (Springer, Berlin, 2003).

[23] Random Rashba fields in InSb depletion layers were reported in M. Morgenstern, A. Georgi, C. Straßer, C. R. Ast, S. Becker, and M. Liebmann, Physica E: Low-dimensional Systems and Nanostructures 44, 1795 (2012).

[24] A. D. Mirlin, D. G. Polyakov, F. Evers, and P. Wölfle, Phys. Rev. Lett. 87, 126805 (2001); A. D. Mirlin, J. Wilke, F. Evers, D. G. Polyakov, and P. Wölfle, Phys. Rev. Lett. 83, 2801 (1999).

[25] A. T. Hatke, M. A. Zudov, J. L. Reno, L. N. Pfeiffer, and K. W. West, Phys. Rev. B 85, 081304 (2012).

[26] S. Faniel, T. Matsuura, S. Mineshige, Y. Sekine, and T. Koga, Phys. Rev. B 83, 115309 (2011) and references therein.

[27] M. Kohda, V. Lechner, Y. Kunihashi, T. Dollinger, P. Olbrich, C. Schönhuber, I. Caspers, V. V. Bel'kov, L. E. Golub, D. Weiss, K. Richter, J. Nitta, and S. D. Ganichev, arXiv:1205.5630. 